\section{Test Set-up to Study the Behavior of a Flexible Instrument in a Bent Tube}

\author{
Jitendra P. Khatait, Dannis M. Brouwer \\ Ronald G.K.M. Aarts, Just L. Herder
}

Mechanical Automation \& Mechatronics
University of Twente, the Netherlands

\section{Background}

The continued involvement of flexible endoscopy in carrying out both diagnostic and therapeutic interventions requires complex manipulation of flexible surgical instrument. The instrument is guided through the endoscope. The motion and force are transmitted to the distal end of the instrument by actuating the proximal end. The friction and the clearance between the instrument and the endoscope, together with the limited stiffness of the instrument, induces non-linear dynamic behavior, which jeopardizes the motion and force fidelity of the instrument.

In order to study the dynamic behavior of the instrument, simulations were carried out using a flexible multibody model. The simulation results showed stick-slip behavior and motion hysteresis, which depend on a number of parameters such as the shape of the bent tube, friction, and stiffness of the instrument.

This paper will discuss the test set-up which was designed to validate the model and simulation results, and also to characterize the instrument behavior inside a bent tube. The design objectives of the set-up are to actuate the instrument tip from the proximal end, to measure the interaction force between the instrument and the bent tube, and to measure the tip motion.

\section{Methods}

The set-up consists of three modules: actuation module (AM), force sensing module (FSM), and tip motion measurement module (T3M). The design specifications of AM are - translation range of $20 \mathrm{~mm}$ with a resolution of 0.1 $\mathrm{mm}$, rotation range of $+/-5$ revolutions with a resolution of 0.5 degree, and translation and rotation speed of $10 \mathrm{~mm} / \mathrm{s}$ and $1 \mathrm{rev} / \mathrm{s}$, respectively. The FSM should have a measurement range of $+/-10 \mathrm{~N}$ with a resolution of $0.02 \mathrm{~N}$. The T3M should be able to do contactless measurement of the tip translation and rotation. The measurement range and resolution of $\mathrm{T} 3 \mathrm{M}$ is in accordance with the actuation.

The AM takes care of actuation of the proximal end in 2DOFs: translation and rotation along the longitudinal axis. Translation is achieved by a combination of a rotational DC motor and a ball screw. The rotational DOF is mounted on a linear slide guide. A flexure coupling - using a bellow and a folded sheet flexure-was designed to connect the two actuated DOFs. A bellow provides a constraint in rotation along the longitudinal axis, while a folded sheet flexure provides a constraint in translation along the axis of fold.

The FSM consists of six 1-DOF load cells. Each load cell is connected to the floating platform through a wire flexure. Force acting along the wire flexure is measured by the attached load cell. It leads to an exact constraint 6-DOFs force measurement. It also includes a preloading mechanism to safeguard the wire flexure and the load cell.

The T3M includes a cam which is supported on a frictionless air bearing through a shaft. The cam is designed for contactless measurement of the translation and rotation of the instrument tip by two laser displacement (LD) sensors.

A bent tube is mounted on the top plate of the FSM. A flexible instrument is inserted through the tube. The AM is aligned to the entry and the $\mathrm{T} 3 \mathrm{M}$ is aligned to the exit. Friction properties can be varied by selecting different materials for the tube and instrument. The stiffness properties of the instrument can be varied by changing the material and cross-section of the instrument.

Figure 1 shows the picture of the assembly of the test setup. All the three modules, together with the key components, are shown in the figure.

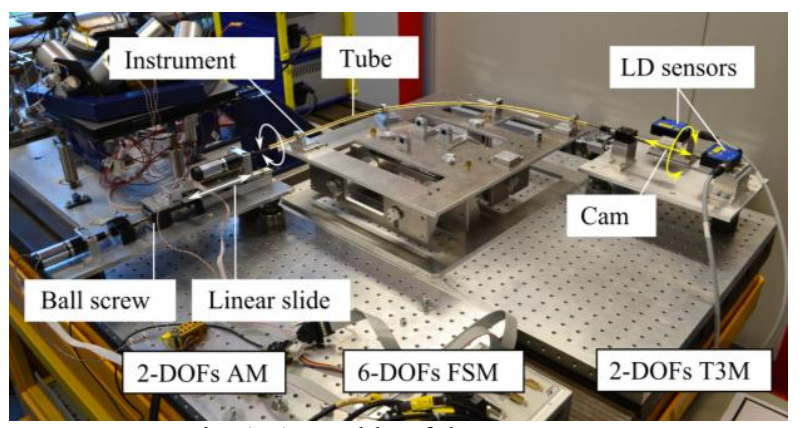

Fig. 1. Assembly of the test set-up

\section{Results}

The AM has actuation range of $50 \mathrm{~mm}$ in translation with a resolution of 0.5 micron. The rotational DOF has no limitation and can rotate with a resolution of 0.09 degree. The AM can achieve a translation speed of $10 \mathrm{~mm} / \mathrm{s}$ and a rotation speed of $1 \mathrm{rev} / \mathrm{s}$ using a gear box.

The load cells can measure up to $+/-100 \mathrm{~N}$. The measurement range is set to $50 \mathrm{~N}$ by preloading. The measurement resolution is $0.015 \mathrm{~N}$ at $1 \mathrm{kHz}$.

For the translation measurement, the LD sensor has a resolution of 25 micron at $1.5 \mathrm{kHz}$ and a measurement range of $50 \mathrm{~mm}$. For the rotation measurement, the LD sensor has a resolution of 10 micron at $1.5 \mathrm{kHz}$ and a measurement range of $20 \mathrm{~mm}$. With the help of the specially designed cam, a resolution of 0.18 degree can be achieved in rotation.

\section{Interpretation}

The test set-up fulfills the design requirements necessary for the study of friction induced dynamic behavior of the flexible instrument inside a bent tube. It also provides a test bench where a scaled-up model of the instrument and tube pair can be studied. The three modules-AM, FSM, and $\mathrm{T} 3 \mathrm{M}$ - enable characterization of the instrument for various configuration of the tube, different friction properties of the interacting surfaces, and stiffness properties of the instrument. The actuation and measurement range, and resolution, of the set-up are higher than the design specifications so that the different aspects can be thoroughly investigated. 